\title{
Recintos sanitarios y espacios de control. Un estudio morfológico de la arquitectura cuarentenaria
}

\section{Quim Bonastra}

(*) Doctor en Geografía Humana, Profesor Asociado, Departamento de Geografía y Sociología, Universidad de Lérida. quim.bonastra@geosoc.udl.cat

Dynamis

[0211-9536] 2010; 30: 17-40
Fecha de recepción: 25 de mayo de 2008

Fecha de aceptación: 8 de diciembre de 2008

SUMARIO 1.--Introducción. 2.-Las necesidades morfológicas del espacio cuarentenario. 3.-El aislamiento del exterior. 4.- La segregación y la purificación de las mercancías. 5.—La compartimentación interior del lazareto. 6.-La vigilancia en el lazareto.

RESUMEN: Tomando como ámbito cronológico el periodo comprendido entre la pandemia de Peste Negra y los inicios de la era bacteriológica y como ámbito territorial Europa, el Mediterráneo y Norteamérica, este artículo se ocupa de la morfología de los lazaretos en su conjunto, teniendo en cuenta las diferentes tradiciones y saberes que influyeron en su configuración

PALABRAS CLAVE: Lazareto, cuarentena, arquitectura, aislamiento, vigilancia, purificación.

KEYWORDS: Lazaretto, quarantine, architecture, isolation, surveillance, purification.

"Les événements de la vie humaine, soit publique, soit privée sont si intimement liés à l'architecture, que la plupart des observateurs peuvent reconstruire les nations ou les individus dans toute la vérité de leurs habitudes, d'après les restes de leurs monuments publiques ou par l'examen de leurs reliques domestiques»

Honoré de Balzac. La recherche de I'Absolu, 1834. 


\section{Introducción $(*)$}

Si, como afirma Balzac, podemos reconstruir las sociedades a través de los restos de sus monumentos públicos, el estudio de las formas de la arquitectura cuarentenaria es, en este sentido, revelador. En el lazareto confluyen y cristalizan una serie de tradiciones y saberes que arrojan mucha luz sobre las sociedades que los construyeron. Si sabemos leer en sus piedras, veremos el nacimiento y la evolución de ciertas ideas sobre la aparición y la difusión de las enfermedades epidémicas. Pero ésta no es toda la información que nos ofrece esta institución; además de las ideas epidemiológicas, y las de otras tradiciones científicas con las que éstas guardan relación, escarbando entre los cimientos del lazareto encontramos todo un muestrario de concepciones políticas, sociales, psicológicas, filosóficas y estéticas que lo configuran, le dan forma y rigen su funcionamiento. Por ello creo necesario un estudio transversal de los espacios cuarentenarios, puesto que se nos presentan como un libro abierto y un campo de pruebas científico, económico, político y social.

\section{Las necesidades morfológicas del espacio cuarentenario}

Los lazaretos eran al mismo tiempo espacios sanitarios, puesto que en ellos se luchaba contra la enfermedad, y espacios de control, dado que esta lucha se realizaba aplicando una lógica carcelaria. En su configuración encontramos, a menudo, elementos formales y de disposición que remiten tanto a hospitales como a cárceles u otros espacios de reclusión, influencias que también podemos hallar en el sentido contrario. Pero antes de analizar estos temas conviene bosquejar las bases sobre las que se asentaba la práctica de las cuarentenas. De estos fundamentos se colegían las diferentes disposiciones adoptadas por los lazaretos que, además, dependían de otros aspectos como las ideas hegemónicas,

(*) Esta investigación se ha financiado, en parte, con fondos del proyecto (SEJ2005-01879/GEOG) del Ministerio de Educación y Ciencia. Presenté una primera versión de este artículo en el congreso «Bicentenario del Llatzeret de Maó: la salud pública ante el peligro epidémico» celebrado en Mahón en septiembre de 2007 en el ámbito de la XVIII Escuela de Verano de Salud Pública. Ciertos aspectos de la argumentación estructurados de manera diferente, aparecen en mi tesis doctoral (Bonastra, Quim. Ciencia, sociedad y planificación territorial en la institución del Lazareto [tesis doctoral]. Universidad de Barcelona; 2006). 
tanto médicas como populares, sobre la generación y el avance de las pestilencias; las modas y cánones arquitectónicos imperantes; o ciertas ideas filosóficas, sociales y morales.

La constatación empírica de la existencia de un periodo de incubación en ciertas enfermedades ${ }^{1}$, que inauguró la era de las cuarentenas, explicaba la aparición de la epidemia en ciudades en cuyo recinto no hubiesen entrado individuos enfermos. Este descubrimiento permitía abolir una defensa contra las pestilencias basada en el cierre total de las comunicaciones de unidades territoriales más o menos extensas y construir una protección que imponía una barrera temporal al tráfico de personas y de mercancías, destinada a retrasar su entrada al lugar en vez de prohibirla. El tiempo de reclusión en el lazareto debía ser, al menos, igual de largo que el que se considerase que duraba la incubación de la dolencia ${ }^{2}$, más el tiempo de precaución considerado necesario por las autoridades de cada lazareto.

Un sistema basado en este principio requería un funcionamiento sin interrupciones, una vigilancia constante del acecho de las epidemias. Por ello, todos los viajeros y mercancías en tránsito debían ser tratados como sospechosos hasta que quedara demostrado que no eran portadores de enfermedades que pudiesen comprometer la salud pública. Así, de manera temprana, se pusieron en marcha mecanismos que atribuían diferentes grados de peligrosidad a las personas y géneros arribados al lazareto, agilizando el cumplimiento de las cuarentenas y asegurando, al mismo tiempo, la defensa contra las epidemias y la continuación del tráfico comercial. Estos dispositivos tenían en cuenta, básicamente, el grado de peligrosidad del lugar de procedencia de los arribados ${ }^{3}$, el tiempo estimado de incubación de la enfermedad en cuestión y la capacidad que tenían las mercancías de retener

1. El primer lugar en el que se aplicaron disposiciones de aislamiento teniendo en cuenta el periodo de incubación fue la República de Ragusa en 1377. Véase Grmek, Mirko D. Le concept d'infection dans l'Antiquité et au Moyen Age, les anciennes mesures sociales contre les maladies contagieues et la fondation de la premiere quarantaine à Dubrovnik (1377). In: RAD. Radovi sa mečunarodnog simpozija u povodu šestote obljetnice dubrovačke karantene. Zagreb: Jugoslavenska Akademija Zantosti i Umjetnosti; 1980, p. 9-54 (p. 49-51).

2. Bonastra, Quim. Ciencia, sociedad y planificación territorial en la institución del Lazareto [tesis doctoral]. Barcelona: Universidad de Barcelona; 2006, p. 121-128.

3. A partir de principios del siglo XVII se afianzó, entre los países cristianos del Mediterráneo, la idea que la peste era permanente en el Levante y en la Berbería. Ver Panzac, Daniel. Quarantaines et lazarets. L'Europe et la peste d'Orient, XVIle-XXe siècles. Aix-en-Provence: Édisud; 1986, p. 31. 
el influjo morbífico ${ }^{4}$. También prestaban atención a ciertas categorías de viajeros considerados extremadamente peligrosos, como los inmigrantes ${ }^{5}$ y los peregrinos ${ }^{6}$.

De lo explicado hasta aquí se desprenden varios corolarios prácticos para la construcción de los lazaretos. Al ser un dispositivo cuyo cometido principal era evitar la importación de epidemias mediante el aislamiento durante un cierto tiempo de los pasajeros y mercancías que pretendían introducirse en una ciudad, el lugar de cuarentena debía ser cerrado, al modo de una fortaleza. Así sería imposible cualquier contacto entre el interior y el exterior que pudiese provocar el avance del contagio. También era importante que en el recinto se aplicara una estricta segregación de las personas y de las mercancías, dada la creencia de que ambos se podían contagiar recíprocamente. Por último, era imperioso evitar que se mezclaran tanto los pasajeros con diferentes grados de peligrosidad, como quienes se encontraran en diferentes estadios de la cuarentena, ya que en ambos casos podían provocarse contagios indeseados. Y lo mismo ocurría con las mercancías.

Aparte de estas líneas generales que, en principio, debían cumplir todos los lazaretos, nos encontramos con una serie de necesidades que era preciso salvar y que dieron pie al diseño de una serie de soluciones diferentes. Su variedad tenía que ver con la época, las ideas médicas, las dimensiones o la importancia del lazareto, el marco social y cultural de su construcción, etc. De este modo, la manera de recibir a los cuarentenistas, el grado de aislamiento, la disposición y aireamiento de sus habitaciones, el almacenamiento y la purga de las mercancías, el ejercicio de la vigilancia, el régimen interno, la intendencia del lazareto o

4. Recordemos las teorías del contagio mediato de Fracastoro: Fracastoro, Girolamo. De sympathia et antipathia rerum liber unus. De contagione et contagiosis morbis et curatione libri III. Venecia: Lucantonio Giunta; 1546. Sobre la limitada originalidad de estas ideas y sobre la recepción de la teoría del contagio de este autor ver, respectivamente: Nutton, Vivian. The seeds of disease: an explanation of contagion and infection from the Greeks to the Renaissance. Medical History. 1983; 27 (1): 1-34; Nutton, Vivian. The reception of Fracastoro's theory of contagion: the seed that fell among thorns? Osiris. 1990; 6: 196-234.

5. Bilson, Geoffrey. «Muscles and health»: health and the Canadian immigrant (1867-1906). In: Mitchinson, Wendy; Dickin McGinnis, Janice, eds. Essays in the history of Canadian medicine. Toronto: McClelland and Stewart; 1988, p. 398-411.

6. Afkhani, Amir A. Defending the guarded domain: epidemics and the emergence of an international sanitary policy in Iran. Comparative Studies of South Asia, Africa and the Middle East. 1999; 19 (1): 122-134. 
su salubridad general, eran cuestiones de cuya resolución se derivaban distintos planteamientos morfológicos.

El estudio de las plantas de numerosos lazaretos permite asociar un buen número de ellos en cuatro grupos. El primero se corresponde con el modelo renacentista, que encontramos en los territorios vénetos y del Milanesado y que estuvo en boga durante los siglos XV y XVI. Estaba basado en una planta claustral de grandes dimensiones y en una separación celular de los internos. Durante el siglo XVIII y comienzos del XIX se desarrolló el modelo ilustrado, que comparte el gusto estético neoclásico y la voluntad comunicativa propia de la arquitectura de aquella época. Tuvo cierto éxito en Italia, Francia y España, aunque la mayor parte de sus exponentes no pasaron del estadio de proyecto. A partir de finales del siglo XVIII se desarrolló el arquetipo de lazareto pabellonario ${ }^{7}$. Se fundamentaba en la multiplicación y en una estricta división de sus unidades constitutivas para favorecer el mantenimiento de una atmósfera sana en todo el recinto. Formulado desde Francia, gozó de bastante aceptación en toda Europa durante el siglo XIX. En la misma época, apareció en Norteamérica el modelo de lazareto paisajista ${ }^{8}$. Su característica fundamental era la diseminación de sus instalaciones en un entorno natural para que los internos recibieran sus efectos sanadores, tanto en lo físico como en lo moral. Sin embargo, muchos lazaretos no se corresponden con ninguno de estos modelos y se tienen que tratar como elementos únicos en su disposición. Por esta razón, me dispondré a desarrollar las principales características morfológicas de los lazaretos en su conjunto, explicando cómo las diferentes necesidades a las que he aludido dieron lugar a formas arquitectónicas concretas.

\section{El aislamiento del exterior}

A menudo, en un esfuerzo por describirlos de manera poética, los lazaretos han recibido calificaciones propias del campo semántico de las cons-

7. Bonastra, Quim. Los orígenes del lazareto pabellonario. La arquitectura cuarentenaria en el cambio del setecientos al ochocientos. Asclepio. 2008; 60 (1): 237-266.

8. Véase Bonastra, Quim. Romanticismo y naturaleza en la prevención de las epidemias en América del Norte. El modelo paisajista de lazareto y su implantación en Canadá. Scripta Nova. Revista Electrónica de Geografía y Ciencias sociales. 2007; 11 (250). Disponible en: http://www. ub.es/geocrit/sn/sn-250.htm. 
trucciones defensivas. Así, se habla de fortalezas sanitarias, de baluartes antiepidémicos, de murallas contra la peste ${ }^{9}$, etc. Estas denominaciones, cuando estudiamos los planos de realizaciones concretas, adquieren un significado especial y relevante, puesto que, en muchos casos, los lazaretos presentan el aspecto de fortalezas que, obviamente, querían evitar el avance de la enfermedad, pero en cuya escenificación debemos ver también una voluntad de comunicar esta idea. En este sentido, el lazareto se presentaba como un lugar de paso entre el mundo exterior - desde el cual acechaba el peligro de la epidemia - y el interior de la ciudad o la zona protegida. Esta carga simbólica tenía una fuerza dramática que no pasó desapercibida ni a sus diseñadores ni a sus observadores ${ }^{10}$.

La incomunicación con el exterior se podía conseguir con el emplazamiento en un lugar apartado o con una disposición en forma de plaza fuerte, siguiendo, en mayor o menor medida, las tendencias de la arquitectura poliorcética. Obviamente, existía una necesidad real de separar el lazareto del exterior, que se apoyaba en diversas teorías epidemiológicas: las que postulaban el contagio inmediato entre individuos; las que contemplaban el mediato a través de efectos capaces de alojar el agente morbífico; las que abogaban por el contagio a distancia ${ }^{11}$; o las que, desechando la idea de contagio, consideraban que la acumulación de enfermos en un lugar podía contaminar la atmósfera de lazareto y ésta, con la ayuda de factores propicios, la del resto de la ciudad ${ }^{12}$.

Los ejemplos de la instalación de lazaretos en islas son numerosos. A modo de lista no exhaustiva podemos citar los de Venecia ${ }^{13}$, el de Corfú,

9. Brusatin, Manlio. II muro della peste. Spazio della pietà e governo del lazzaretto. Venezia: Cluva; 1981; Vanzan Marchini, Nelly-Elena. Rotte mediterranee e baluardi di sanità. Venezia e i lazzaretti mediterranei. Milano: Skira; 2004; Vidal Hernández, Josep Maa. El Llatzeret de Maó, una fortalesa sanitària. Mahón: Institut Menorquí d'Estudis; 2006.

10. «Fu fatto l'anno 1468, un'altro Lazaretto chiamato Nuovo per esser posteriore in fabrica al Vecchio, con cento camere, e una vigna serrata, il quale dalla lontana ha sembianza di castello, come amplo di circuito». En Sansovino, Francesco. Venezia città nobilissima e singolare (1581). Venecia: Filippi Editore; 1968, p. 232.

11. Nutton, n. 4, 1990, p. 200.

12. Como en d'Agramont, Jacme. Regiment de preservació a epidímia o pestilència e mortaldats. 1348, fol. 3r. Véase García Ballester, Luis; Arrizabalaga, Jon. El regiment de Jacme d'Agramont y el Estudi de Medicina de Lleida. In: d'Agramont, Jacme. Regiment de preservació de pestilencia. Barcelona: Enciclopedia Catalana; 1998.

13. Ante la imposibilidad de incluir planos, alzados o vistas de todos los lazaretos que se citarán en este texto remito al lector al Apéndice Gráfico no 1 de mi tesis doctoral, que se puede consultar en: http://tdcat.cesca.es/TDX-0728106-122030/index.html. 
los canadienses de la Grosse-île (en Quebec) y de Partridge Island (en Nueva Brunswick), el marsellés Hôpital Caroline, el de San Simón (en Vigo) o, si hacemos un cambio de escala, el lazareto de Mahón, que era insular respecto a España ${ }^{14}$. De todos modos, los lazaretos debían colocarse en lugares que, además, debían ser prácticos para el desempeño de las funciones que les eran propias y permitir, en el caso de los lazaretos marítimos, el fondeo sin embarazo.

Por ello, en muchas ocasiones, ante la imposibilidad de encontrar un lugar suficientemente aislado, se optaba por el emplazamiento en sitios más cercanos a la ciudad. Si el lugar lo permitía, se tomaban otro tipo de precauciones, como la instalación del lazareto en parajes situados de tal manera respecto a la ciudad, que los vientos dominantes se llevaran cualquier agente pestilencial emanable de él ${ }^{15}$.

Pero muchas veces, los lazaretos se localizaban a las puertas mismas de la ciudad, ocupando un espacio cercano a sus murallas o estrechamente relacionado con el puerto. Entonces, las estaciones cuarentenarias se presentaban como una fortaleza, con toda su carga simbólica. Lo vemos claramente en ciudades del Adriático oriental, como Split o Ragusa, en las cuales el lazareto ocupaba un puesto privilegiado en el puerto. Aunque se hace más evidente en el lazareto de San Rocco de Liorna y en Ancona. En el primer caso, la estación de cuarentena se encontraba del todo imbricada en el complejo defensivo de la ciudad, ocupando el interior de un hornabeque de la muralla que la rodeaba por la parte de tierra. En el lazareto de Ancona, obra de Luigi Vanvitelli, el deseo de comunicar la idea de fortaleza alcanza el nivel más alto, puesto que, a la manera de las ciudadelas renacentistas, el edificio presenta una planta pentagonal. Para reforzar la carga simbólica del edificio, apuntando al mar, se construyó, incluso, un baluarte ${ }^{16}$. Lo mismo ocurre con el proyecto de

14. Vidal Hernández, Josep Maria. El lazareto de Mahón y la sanidad en los puertos españoles a principios del siglo XIX. In: Morales Moya, Antonio, coord. 1802. España entre dos siglos. Ciencia y Economía. Madrid: Sociedad Estatal de Conmemoraciones Culturales; 2003, p. 119-136.

15. Hély d'Oissel, Abdon-Patrocle. Rapport sur l'établissement de nouveaux lazarets, adopté par la commission sanitaire formée près le ministère de I'Intérieur. París: Imprimerie Royale; 1822, p. 14.

16. Mezzetti, Carlo; Bucciarelli, Giorgio; Pugnaloni, Fausto. Il lazzaretto di Ancona, un'opera dimenticata. Ancona: Cassa di Risparmio di Ancona; 1978, p. 102-143. 
lazareto para Barcelona, firmado por Joaquín Rigalt en $1821^{17}$, que parece inspirado en el romano Castel Sant'Angelo ${ }^{18}$.

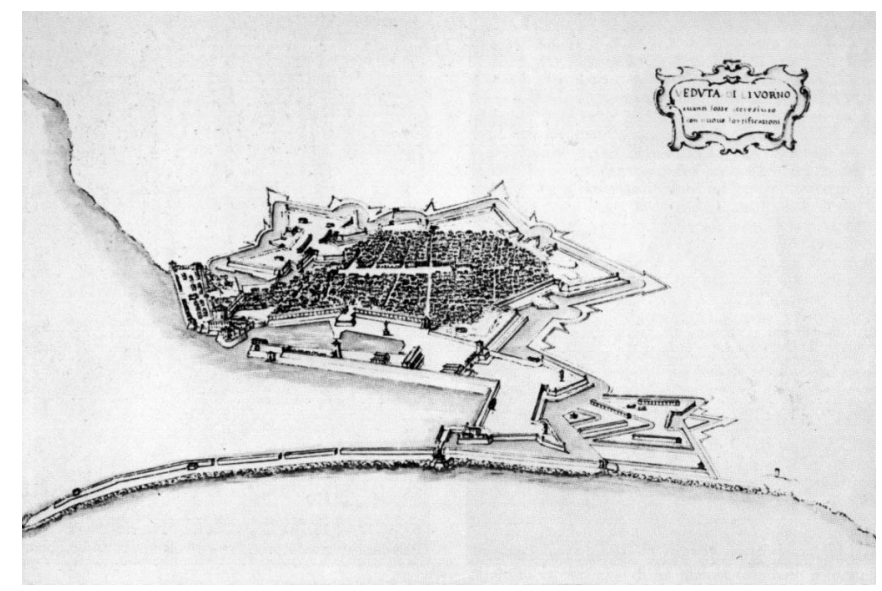

Figura 1: Vista de la ciudad y las fortificaciones de Liorna a finales del siglo XVII. En primer término se puede observar el hornabeque de la muralla que albergaba el lazareto de San Rocco. Fuente: Istituto Storico e di Cultura dell'Arma del Genio Militare, cart XXII, número 1630.

Casi todos los edificios cuarentenarios contaban con un muro de cinta que, en mayor o en menor medida, les confería un aspecto de fortificación. En ciertos casos esta sensación se veía reforzada por la existencia de torreones de vigilancia ${ }^{19}$ o por la colocación de un foso alrededor de la pared del lazareto ${ }^{20}$.

Como vemos, el lazareto debía transmitir la imagen de que era una fortaleza inexpugnable capaz de parar los ataques de las pestilencias, pero en la

17. Sus planos se conservan en el Arxiu Històric de la Ciutat de Barcelona: Rigalt, Joaquín. Proyecto de un Lazareto Público o casa de sanidad. Barcelona: 1821. Localizado en: ACHB, PL-4.2-728, y Rigalt, Joaquín. Plano geometría de la Casa de Sanidad o Lazareto público cuya fachada se demuestra en el diseño nº. 2. Barcelona: 1821 Localizado en: AHCB, PL-5.5-1263.

18. En este caso las referencias simbólicas son dobles, puesto que el nombre del Castel Sant'Angelo, como explica Jacobus de Varagine en su Legenda Aurea (Légende dorée (s. XIII). París: Perrin et Cie.; 1910, p. 169, está relacionado con la epidemia de peste que sufrió Roma en 590 d.C.

19. Por ejemplo, los que encontramos en las Nouvelles Infirmeries de Marsella, en el lazareto de Corfú o en el de Cefalonia.

20. Encontramos buenos ejemplos en Milán y en Liorna (San Rocco y San Leopoldo). 
práctica tenía que defender a la ciudad de las enfermedades que albergara. Es por ello que muchos de los diseños utilizados son construcciones abiertas hacia un patio interior, como todos los que siguen el modelo renacentista basado en el claustro ${ }^{21}$, casi todos los del adriático oriental ${ }^{22} \mathrm{o}$ algunos de los construidos en el mar Egeo ${ }^{23}$.

Los lazaretos que no se orientaban claramente hacia el interior, como el de Génova, solían tener un muro de cinta alrededor de sus edificios. Algunos, presentaban una doble línea de murallas que servía a la vez como camino de ronda para los vigilantes y como cinturón preventivo de aire que ayudaría a evitar los posibles contagios e infecciones a distancia. Donde esta idea se encuentra más desarrollada es en el modelo de lazareto en pabellones, en el que estos asuntos representaban una preocupación especial. Un exponente temprano de este modelo fue el lazareto de Mahón ${ }^{24}$.

Se trataba, en definitiva, de construir un espacio cerrado, sujeto a una normativa específica, la cuarentenaria. No era una simple barrera que impidiese el paso de la epidemia, sino el lugar del cual solamente se podía salir sano, purificado. En este sentido, el lazareto debía ejercer un efecto disuasorio de cara al exterior, erigiéndose como un bastión inexpugnable frente a cualquier epidemia. Tenía que comunicar que la ciudad era una escala sana que se defendía contra las epidemias y que no era fácil burlar su vigilancia. Esto se conseguía con el efecto teatral de su arquitectura, que avisaba a quienes se acercaban que no se podrían sustraer al control que ejercía sobre barcos, mercancías y pasajeros, pero también, de manera más sutil, con el rigor del trato dispensado a los sospechosos y con la ejemplaridad de las penas pronunciadas contra quienes quebrantaran su reglamento, que serían debidamente publicitadas ${ }^{25}$. Al mismo tiempo, de cara a los moradores de la ciudad

21. Por ejemplo, el lazareto de Milán, el Nuovo de Venecia, el de Verona o el de Bérgamo.

22. Por ejemplo, el lazareto de Ragusa y los venecianos de la Dalmacia y las islas Jónicas: Split, Castelnuovo, Corfú, Cefalonia y Zante.

23. Por ejemplo, los lazaretos de Sira y Samos.

24. «Debe estar circuído de dos paredes muy altas (lo menos de 9 varas y media paralelas, que hacen una doble cerca que dexa un bacío, o camino en medio de 14 a 15 varas, para que nada se pueda tirar de dentro a fuera, y para otras precauciones». Fernández de Angulo, F. Ydea del proyecto de un lazareto general de cuarentena en el puerto de Mahón ysla de Menorca... 1786. Localizado en: Servicio Histórico Militar (Madrid), exp. 12.892, sig. 3-3-1-5.

25. Hildesheimer, Françoise. Le Bureau de la Santé de Marseille sous l'ancien régime: le renfermement de la contagion. Marsella: Fédération historique de Provence; 1980, p. 215-216. 
donde estuviera emplazado, debía tener un efecto balsámico y actuar como tranquilizador de sus ánimos ${ }^{26}$.

\section{La segregación y la purificación de las mercancías}

En algunas ocasiones, la práctica de ciertas operaciones precede a la consolidación o, incluso, a la enunciación de una teoría que las explica. La práctica de las cuarentenas y, en general, todo el sistema cuarentenario estaban fundados en un cuerpo de conocimientos dentro del cual ocupaban una situación central los saberes médicos hegemónicos en cada periodo. De todos modos, al tratarse de un establecimiento ideado, diseñado y dirigido por administradores públicos, que no pertenecían necesariamente al gremio médico, este cuerpo de conocimientos se veía ampliado y matizado por todo el poso cultural, moral y vivencial de la sociedad en la que se enmarcaba, por lo que ciertas coyunturas económicas, políticas y sociales también desempeñaban un papel muy importante en todo ello ${ }^{27}$. Esto explica, por ejemplo, la temprana separación de mercancías y pasajeros en los primeros lazaretos, bastante antes de la exposición de la teoría del contagio mediato por parte de médicos como Michele Savonarola, Alessandro Benedetti o, más tarde, Girolamo Fracastoro. Si bien es obvio que, para el nacimiento y el desarrollo del sistema cuarentenario hacía falta una aprehensión del fenómeno epidémico en el cual el contagio fuera la piedra angular, no parece descabellado proponer que la temprana práctica de segregar individuos y mercancías pudiese venir, por ejemplo, de una asociación entre el mal olor, o los olores fuertes, y las pestilencias, consideradas por algunos una corrupción del aire ${ }^{28}$. Así, era fácil que ciertos géneros que presentaran un fuerte olor, como la lana sin

26. «Cette population de Marseille si impressionnable, qui craint tant la peste, ne s'émeut pas le moins quand on lui dit que la peste est au lazaret, tant elle compte sur l'expertise des agents de la santé». Ségur-Dupeyron, P. de. Rapport adressé à son Exc. le Ministre du Commerce, par..., Secrétaire du Conseil de Santé, chargé de procéder a une enquête sur les divers régimes sanitaires de la Méditerranée. París: Imprimerie Royale; 1834, p. 28.

27. Bonastra, n. 2, p. 482-484.

28. Arrizabalaga, Jon. Facing the Black Death: perceptions and reactions of university medical practitioners. In: García Ballester, Luis et al., eds. Practical medicine from Salerno to the Black Death. Cambridge: CUP; 1994, p. 237-288. 
tratar o los cueros, fueran considerados vehículos del contagio, y más aún si provenían de lugares sospechosos.

El problema de partida es cómo se planteaba la separación de las mercancías y qué espacio debían ocupar en el seno del lazareto. Una segunda cuestión, aunque no menos importante, es el trato que recibían estos géneros, dado que su purificación también tenía repercusiones espaciales. El factor fundamental era el grado y el número de días en que las diferentes mercancías eran capaces de contener el agente pestilencial.

Respecto al lugar que estos productos ocupaban en el recinto cuarentenario nos hallamos, de nuevo, ante una variedad de prácticas. En todos los lazaretos encontramos lugares especiales para el almacenamiento y la purga de las mercancías. Su disposición, medidas u otras características variaban según la importancia que se le diera a estos procedimientos, o la jerarquía y dimensiones del edificio. A veces, los géneros de un barco ocupaban la planta baja de una construcción y el piso elevado se utilizaba para albergar a sus pasajeros o tripulantes ${ }^{29}$, en otros ocupaban habitaciones contiguas del mismo edificio ${ }^{30}$, en otros casos ocupaban espacios totalmente separados ${ }^{31}$ y sin ninguna posible comunicación entre ellos. Algunas ciudades se dotaban de edificios totalmente separados y situados en partes diferentes para alojar a mercancías y tripulantes ${ }^{32}$.

Uno de los elementos principales del proceso de purificación era el oreo de las mercancías que, dependiendo de su fragilidad, podía hacerse al aire libre o a cubierto en los almacenes. Para ello los almacenes debían estar lo suficientemente abiertos para permitir la entrada y la salida del aire. Esto se conseguía de maneras diversas, como la colocación de ventanas en ambas paredes para conseguir una corriente continua de aire ${ }^{33}$, la utilización de un cobertizo abierto completamente por alguno de sus lados ${ }^{34}$.

\footnotetext{
29. Por ejemplo, en el lazareto de Ragusa o en el de Castelnuovo.

30. Por ejemplo, en el Lazaretto Vecchio de Venecia y en el de Ancona.

31. Son un buen ejemplo de ello los tres lazaretos de Liorna.

32. En Verona, el sborro estaba dedicado a las mercancías y el lazareto en forma de claustro consagrado a los viajeros.

33. Por ejemplo, en los almacenes del lazareto de Génova. Véase Howard, John. An account of the principal Lazarettos in Europe, with various papers relative to the plague and additional remarks on the present state of prisons in Great Britain and Ireland. 2nd ed. Londres: Johnson, Dilly and Cadell; 1791, p. 6.
}

34. Práctica utilizada en los tres lazaretos de Liorna o en el Zante, que describe someramente Howard, n. 33, p. 10. En el Lazzaretto Vecchio de Venecia se combinaban ambos tipos de cobertizo. Véase An Authentick Account of the Measures and Precautions used at Venice, 


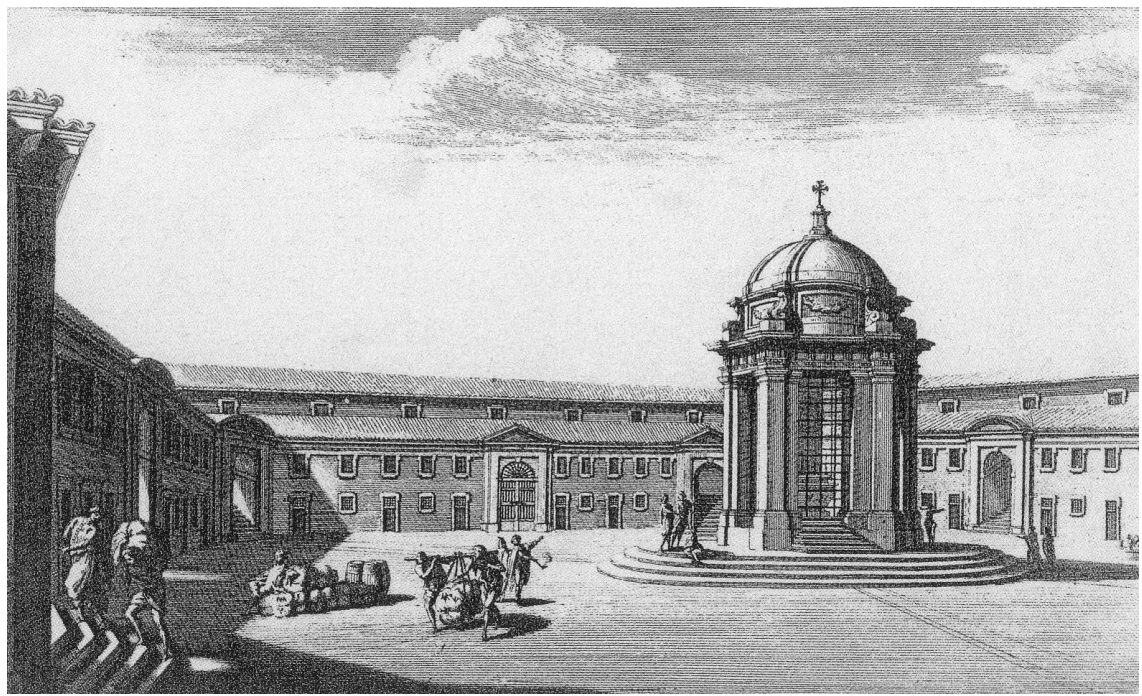

Figura 2: Vista del patio interior del lazareto de Ancona en el siglo XVIII.

Fuente: Giuseppe Vassi, Biblioteca Apostolica Vaticana, GDS, Stampe, Capponi II. 16, fig. 28.

O la disposición de de almacenes abiertos por los cuatro lados para dejar pasar el aire libremente ${ }^{35}$.

Los materiales más porosos, como la lana, el algodón o las telas se suponían más susceptibles de poder albergar la influencia morbífica. Esto hacía que, en muchas ocasiones, antes de ser admitidos en el lazareto, fueran sumergidos en el mar. De ahí solían pasar a algún patio para pasar la cuarentena a sol y al sereno. Periódicamente, los mozos de carga del lazareto abrían los fardos y los giraban para que recibiesen el baño del sol y de aire puro por todos los lados ${ }^{36}$. Todas estas operaciones, en conjunto, debían asegurar su purificación. Los materiales más frágiles se trataban con más cuidado y solían, cuando las instalaciones del lazareto lo permitían,

by the magistrate of the office of health, for the preservation of the public health. Londres: Edward Owen; 1752, p. 14.

35. Lo vemos en numerosos lazaretos. En las Nouvelles Infirmeries de Marsella servían para colocar las mercancías de valor. Véase Howard, n. 33, p. 5.

36. Para ello existían en algunos lazaretos recintos especiales con bancos sobre los cuales se dejaban los fardos de algodón o de lana para que no se ensuciasen. Véanse como ejemplo los de Marsella, Malta o Mahón. 
almacenarse en tinglados abiertos por los costados, para dejar correr el aire. Los animales también eran objeto de purificación. A los de pelo se les podía someter a un pediluvio o conducirlos a través de un canal lleno de agua. A los de pluma se les podía rociar con vinagre ${ }^{37}$. Estas operaciones dependían, obviamente, de los materiales que en cada momento y lugar se consideraran más peligrosos.

Además de todo ello, también era importante que las mercancías, durante la purga, no contagiasen a los internos, lo que se conseguía con una estricta separación de ambos tipos de huéspedes. La asociación entre los olores fuertes y la putrefacción del aire que, según ciertas teorías epidemiológicas, estaba en el principio de las pestilencias hacía que, por un lado, se practicaran técnicas de purificación con sustancias olorosas, sahumerios y fumigaciones ${ }^{38}$ y que, por otro, se asignara a ciertos materiales, a causa de su pronunciado olor, un recinto propio para la cuarentena. Este era el caso, por ejemplo, del cercado de los cueros en las Nouvelles Infirmeries de Marsella, situado a unos centenares de metros del complejo.

Esta primera clase de segregación, la que se ejercía sobre las mercancías, era subsidiaria y complementaria de aquella que se operaba sobre los viajeros. En el apartado siguiente mostraré cómo ambas segregaciones encajaban en el interior del lazareto y cómo la aplicación de diferentes ideas sobre el encierro cuarentenario supuso distintos niveles de separación y compartimentación del interior del lazareto.

\section{La compartimentación interior del lazareto}

La eficiencia del sistema cuarentenario estaba basada en la consideración de que todos los viajeros en tránsito eran posibles portadores de la pestilen-

37. Viallon-Schoneveld, Marie. Et Venise inventa le lazeret ou les los sanitaires contre la peste. In: Viallon-Schoneveld, Marie, ed. Médecins et médecine au XVle siècle. Saint-Étienne: Publications de I'Université de Saint-Étienne; 2002, p. 198-213.

38. Lo que nos lleva a las diferentes concepciones sobre la naturaleza del elemento contagiante y, por consiguiente, del modo de destruirlo. Para este debate en España de finales del siglo XVIII y comienzos del XIX, véase Carrillo, Juan Luis; Riera Perelló, Pedro y Gago, Ramón. La introducción en España de las hipótesis miasmáticas y prácticas fumigatorias. Historia de una polémica (J.M. Aréjula-M.J. Cabanellas). Medicina e Historia. 1977, (67): 8-26; Bertomeu, José Ramón; García Belmar, Antonio. La revolución química. Entre la historia y la memoria. Valencia: PUV; 2006, p. 211-230. 
cia. Ello significaba que debía funcionar de manera continuada y tenía que mantenerse vigilante e informado del estado de salud del resto del mundo en todo momento. Esta categorización del sospechoso y la constatación de que no todos los viajeros lo eran en el mismo grado tuvieron, lógicamente, repercusiones morfológicas en el interior del lazareto.

Si existían áreas afectadas por la peste de manera permanente, zonas temporalmente sospechosas y regiones sanas, por citar algunas de las coyunturas posibles, los barcos, viajeros y caravanas que arribaban desde cada una de ellas debían contar con espacios diferenciados en el lazareto. Sus huéspedes, para evitar el contagio, debían ser sometidos a una estricta compartimentación en función del grado de peligrosidad que representaran.

En los primeros lazaretos importantes, erigidos en los siglos XV y XVI, nos encontramos, en general, con soluciones arquitectónicas fundadas en la repetición de espacios independientes de cuarentena. Se podían añadir o sustraer módulos de encierro sin que, por ello, resultara alterada la manera de llevar a cabo la cuarentena ni el funcionamiento general del complejo. El ejemplo paradigmático de este tipo de lazareto es el Vecchio de Venecia, que presenta una superposición de módulos de cuarentena totalmente aislados los unos de los otros ${ }^{39}$. Cada uno de estos encierros estaba compuesto por una entrada directa desde el exterior, un gran almacén para las mercancías, apartamentos separados para diferentes grupos de cuarentenistas y un patio de aireación relativamente extenso. De este modo, cada una de estas unidades funcionaba como un lazareto separado y, si en el interior de una de ellas se manifestaba la peste, las demás podían seguir operando normalmente.

El lazareto de Ragusa estaba construido siguiendo la misma idea de recintos separados en serie. Se trataba de cinco espacios, con una puerta de entrada desde el mar y una de salida hacia tierra. Cada espacio tenía un patio central hacia el que se abrían las arcadas de los almacenes para las mercancías situadas a los lados. Encima de cada tinglado se encontraba la estancia para los pasajeros de un barco de modo que pudieran realizaran la cuarentena de manera separada y sin posibilidad de mezclarse ${ }^{40}$.

En los lazaretos del modelo renacentista el edificio funcionaba como un todo orgánico al que no se podía amputar ningún miembro. Las cámaras

\footnotetext{
39. An authentick account ..., n. 34, p. 14.

40. Janeković Römer, Zdenka. I lazzaretti de Dubrovnik (Ragusa). In: Vanzan Marchini, n. 9, p. 246249.
} 
seriadas para los cuarentenistas se disponían, en forma de claustro porticado, alrededor de un patio común, que cumplía las funciones de lugar de almacenamiento y de espacio abierto para la aireación de las estancias y de las mercancías. En algunos de estos lazaretos empezamos a encontrar los primeros intentos de segregación funcional de los internos. En Milán, aparentemente, se separaba a hombres y a mujeres y, además, había habitaciones diferentes para médicos, sospechosos, enfermos y convalecientes ${ }^{41}$, aunque esta separación era meramente nominal y no física. En el mismo orden de cosas, en el lazareto de Verona se empezó a aplicar un tipo de separación dependiendo del lugar de procedencia de los viajeros, creándose en el patio una separación real mediante muros que dividían el recinto en cuatro y anticipaban el sistema de departamentos. A cada uno de los recintos que creaba esta división interior se entraba y se salía por una puerta distinta, que se situaba en el punto central de cada uno de los cuatro muros ${ }^{42}$.

A partir del siglo XVI se empezó a aplicar el sistema de patentes sanitarias y, paralelamente, ciertos lazaretos comenzaron a utilizar en su organización el sistema de separación en departamentos. Éste se correspondía, en mayor o menor medida, con las principales clases de patente sanitaria. Un ejemplo temprano es el inmenso lazareto de Génova, que contaba con dos departamentos separados y aislados entre sí, el sucio y el sospechoso. Cada uno de ellos consistía en un gran patio cuadrangular alrededor del cual se alzaban, ocupando dos lados de cada patio, las habitaciones para los pasajeros y las mercancías correspondientes a cada patente. En el interior de cada edificio encontramos habitaciones seriadas destinadas a grupos más o menos pequeños de cuarentenistas que hubiesen llegado en el mismo barco. El edificio central del lazareto, que servía de separación entre ambos departamentos, estaba ocupado por la administración y por almacenes reservados para los bienes ya purificados. Toda la parte del lazareto orientada hacia tierra estaba sin edificar para permitir el aireamiento de los edificios ${ }^{43}$.

41. Patteta, Luciano. Nuove ipotesi sul Lazzaretto quattrocentesco di Milano. Bollettino d'Arte. 1986; 35-36: 25-42 (32).

42. Camerlengo, Lia. Il lazzaretto a San Pancrazio e l'ospedale della Misericordia in Bra. Le forme dell'architectura. In: Pastore, Alessandro et al. L'ospedale e la città. Cinquecento anni d'arte a Verona. Verona: Cierre Edizioni; 1996, p. 179-191.

43. Howard, n. 33, p. 5-7. 
Esta división en departamentos se fue repitiendo a partir de ese momento en bastantes de los lazaretos construidos de nueva planta. En el conjunto cuarentenario de Liorna esta idea se llevó al límite. En la Edad Moderna, esta ciudad, que era el puerto franco del Gran Ducado de la Toscana, se vio obligada ampliar sucesivamente sus dependencias cuarentenarias a causa del gran tráfico marítimo que soportaba ${ }^{44}$. Así, al lazareto de San Rocco, construido en la entrada del puerto a finales del siglo XVI, se le añadió, a mediados del siglo siguiente, el complejo de San Jacopo, situado a dos kilómetros al sur. Éste funcionaría, a partir de entonces, como departamento de patente sucia. Tras diversas ampliaciones de este recinto se decidió construir, en el último tercio del siglo XVIII, el lazareto de San Leopoldo ${ }^{45}$, que daría forma definitiva al complejo de defensa antiepidémica del puerto y que estaba situado al sur de sus predecesores. El lazareto de San Leopoldo hacía las veces de departamento de patente sucia, mientras que los otros dos estaban consagrados a la patente sospechosa y a la limpia respectivamente. Además, los tres recintos estaban unidos por un canal por el cual se transportaban las mercancías expurgadas. A pesar de que esta disposición resultara de la imposibilidad casi total de aumentar substancialmente el tamaño del lazareto de San Rocco y, más tarde, del de San Jacopo, sin comprometer sus operaciones, el resultado final resulta muy interesante. Su diseño representa una evolución de la idea de compartimentar al máximo el interior del lazareto teniendo en cuenta la peligrosidad sus huéspedes. De hecho, esta práctica se utilizaba, aunque quizás de una manera menos clara, en algunas de las ciudades que, al igual que Liorna, contaban con una multiplicidad de establecimientos cuarentenarios. Es el caso de Marsella, que durante algunas décadas del siglo XIX utilizó las instalaciones de tierra de las Nouvelles Infirmeries, los nuevos edificios insulares del Hôpital Caroline y el desembarcadero y resto de almacenes de las islas del Frioul.

Este aislamiento de las diferentes partes del interior del lazareto alcanzó sus cotas más importantes de desarrollo, tanto a nivel teórico como morfológico, en los lazaretos en pabellones. Se trata de los primeros laza-

44. Cipolla, Carlo M. II burocrate e il marinaio. La «Sanità» toscana e le tribolazioni degli inglesi a Livorno nel XVII secolo. Bolonia: II Mulino; 1992. Ciano, Cesare. La sanità marittima nell'età medicea. Pisa: Pacini Editore; 1976.

45. Martines, Vincenzo. II lazzaretto de San Leopoldo e di San lacopo di Livorno. In: Vanzan Marchini, n. 9, p. 286-291. 
retos en los que encontramos una voluntad de crear un modelo fundado sobre bases científicas y en los que se llevó a cabo un trabajo previo de definición de las necesidades, de evaluación de las posibles contingencias y de proposición de formas y disposiciones que deberían servir de modelo al nuevo tipo de infraestructuras cuarentenarias. Aunque debemos dar la preeminencia en la formulación del nuevo tipo de lazaretos al esfuerzo realizado por médicos, ingenieros y arquitectos franceses a lo largo de unas cuantas décadas a caballo entre los siglos XVIII y XIX, no debemos olvidar los trabajos realizados por profesionales de otros países que contribuyeron a su enunciación y desarrollo ${ }^{46}$.

Tomando como base el ejemplo del pensamiento nosológico, que siguiendo el patrón de la botánica agrupaba las enfermedades por especies, en el lazareto - como estaba ocurriendo también en el hospital- se desarrollaron al máximo las tareas de clasificación y, por tanto, de división de los espacios con el fin de prestar a los recluídos una atención más efectiva y ordenada y de evitar la propagación del contagio. Cada pasaje debía tener sus apartamentos, cada cargamento su espacio para la purificación; todo ello debía estar bien separado y ordenado en el interior del departamento que les correspondía. Además, el aire del recinto debía mantenerse puro para evitar que el exhalado por los enfermos o las mercancías pudiera afectar al resto de confinados. Para ello, las subdivisiones se multiplicaban al máximo, con una profusión de pabellones y vallados dispuestos de tal manera que permitiesen la organización de los trayectos y operaciones que se desarrollaban en el interior del recinto y el mantenimiento de una atmósfera sana en todo el complejo ${ }^{47}$. Aparte de la multitud de proyectos que proponían soluciones formales en este sentido ${ }^{48}$, los principales lazaretos construidos siguiendo este modelo fueron el de Mahón, el Hôpital Caroline de Marsella, el lazareto Marie-Thérèse de Burdeos y el de Mindin, en la desembocadura del Loira.

\footnotetext{
46. Véase Bonastra, n. 7, p. 237-266.

47. Morachiello, Paolo. Bruyère, Comacchio e il «programma» dei Lazzaretti in Italia (1805-1823). Casabella. 1978; 439: 52-59.

48. El de John Howard para Inglaterra, el de Pompeo Schiantrelli para Mesina, los de Bruyère para Comacchio, los de Alavoin y Godde para Francia, el de Giuliano de Fazio para Nápoles o el de William Marsden para Quebec. Sobre este último, véase Bonastra, Quim. La cuarentena en Quebec y el plan preventivo de William Marsden (1832-1866). Scripta Nova. Revista Electrónica de Geografía y Ciencias Sociales. 2005; 9: 195. Disponible en: http://www.ub.es/geocrit/sn/sn195.htm.
} 


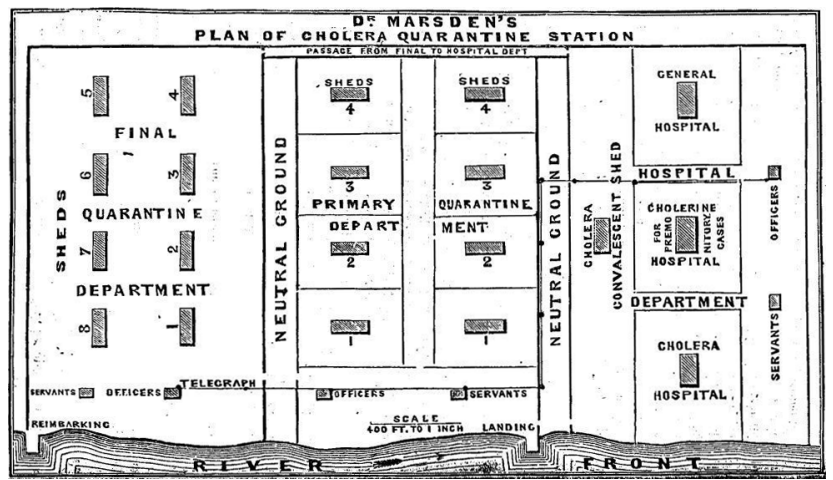

Figura 3: Proyecto de lazareto en pabellones para la provincia de Quebec a mediados del siglo XIX. Fuente: Marsden, William. Plan of Quarantaine for Cholera. Canada Medical Journal and Monthly Record of Medical and Surgical Science, 1866; 2, p. 338.

En Norteamérica se desarrolló, a partir de finales del siglo XVIII, un modelo de lazareto que poco tenía que ver con los europeos. El contexto histórico en que surgió era el de las importantes corrientes migratorias entre Europa y el Nuevo Mundo. En su configuración encontramos un conjunto de concepciones científicas, morales y estéticas que mezclaba los idearios romántico, naturalista, paisajista y protestante, que cristalizaban en la afirmación siguiente: para conseguir un entorno saludable para los huéspedes del lazareto ${ }^{49}$ se tenía que reproducir en su seno un ambiente lo más parecido posible a la naturaleza ${ }^{50}$. Este entorno natural debía aportar a los inmigrantes una serie de beneficios físicos y morales que les prepararían para su entrada en el país. Esta idea adquiere aún más importancia si tenemos en cuenta la idea predominante en Norteamérica de que estas oleadas de inmigrantes eran el excedente social de los países europeos.

A nivel funcional, el lazareto de corte paisajista era una evolución del lazareto pabellonario, aunque siguiendo los postulados románticos. De hecho se correspondería con el modelo de hospital pabellonario disperso ${ }^{51}$.

49. Al revés de lo que pasaba en los lazaretos europeos, que solían estar consagrados a la purificación de mercancías y dejaban un espacio relativamente reducido para los pasajeros de los barcos, los lazaretos americanos solían albergar, sobre todo, inmigrantes.

50. Estas ideas se desarrollan más extensamente en Bonastra, n. 8.

51. Cabal, Michel. Hôpitaux: corps et âmes. París: Rempart; 2001. Vogel, Morris J. The transformation of the American hospital. In: Norbert Finzsch and Robert Jütte, eds. Institutions of confine- 
Construido a imagen de un pueblo bucólico y desordenado - en el que se encontraban esparcidas todas las funciones necesarias para el ejercicio de las operaciones de cuarentena- perdía la compartimentación interior, al menos la física, en forma de altos muros que evitaban tanto el contacto visual como el corporal. La circulación interior se realizaba por sinuosos caminos en un entorno natural agradable y salutífero, y la amplitud de sus instalaciones podía, de algún modo, evitar los encuentros no deseados, tal como ocurría en los lazaretos pabellonarios.

\section{La vigilancia en el lazareto}

Hasta aquí hemos visto el lazareto como un espacio acotado, separado del exterior, sujeto a las normas cuarentenarias, compartimentado y ordenado en su interior para evitar la propagación del contagio. Naturalmente, esto no podía conseguirse sin una estricta vigilancia. En este punto, el lazareto, que se ha revelado como un recinto sanitario y auxiliar de la economía, confluye con las instituciones penitenciarias, con las que comparte muchas características, tanto morfológicas como de funcionamiento. Estaba sujeto a la jurisdicción exclusiva de los intendentes sanitarios que ejercían un control absoluto sobre todo cuanto sucedía en el interior del recinto, imponiendo los días de cuarentena, examinando la purificación de las mercancías y fiscalizando el comportamiento de los confinados. Para ello se necesitaba la aceptación de la dictadura ejercida por los intendentes de sanidad en el lazareto ${ }^{52}$, conforme a unos supuestos que permitían el recorte de parcelas de libertad a favor del bien común ${ }^{53}$.

Un primer ámbito de vigilancia se ejercía hacia el exterior. Se debía evitar a toda costa que los internos comunicaran con el exterior del recinto. Esto se conseguía con muros ciegos, fosos, torres de vigía y la instalación

ment. Hospitals, asylums, and prisons in Western Europe and North America, 1500-1950. Cambridge: CUP; 1996, p. 39-54.

52. Por ejemplo, el prior del lazareto de Venecia, mientras estaba de servicio, no estaba sujeto ni a la jurisdicción civil ni a la criminal, solamente a la de los provveditori di sanità. Howard, n. 33, p. 15.

53. Estos supuestos eran, según Hildesheimer: el miedo a la epidemia, el secreto de las operaciones que se desarrollaban en el lazareto, «auxiliar ideal del poder absoluto», y la ejemplaridad en la imposición de las cuarentenas y en el trato dispensado a los que la quebrantasen. Ver Hildesheimer, n. 25, p. 215-216. 
del lazareto en lugares poco accesibles. En muchos de ellos - sobre todo en los pabellonarios - el muro de cinta se torna doble, para dar más espacio a la ventilación, y para la ronda de los guardias. En algunos casos, alrededor de esta doble muralla se colocaba, además, un camino de ronda ${ }^{54}$.

Asegurado el aislamiento del exterior, convenía prever la vigilancia interior. Obviamente, la visión del lazareto desde el exterior, tenía que actuar como elemento disuasorio de las conductas inadecuadas. John Howard, en su informe sobre los lazaretos del Mediterráneo, ya advertía que, en muchos casos, los lazaretos tenían demasiado el aspecto de prisiones, cosa que atemorizaba a los que debían cumplir la cuarentena ${ }^{55}$. A pesar de todo ello, convenía mantener un estricto control sobre los pasajeros, a quienes se obligaba a mantener un orden que, en algunos casos, no distaba mucho del impuesto en las instituciones carcelarias.

Este control sobre el lazareto se practicaba adoptando soluciones muy variadas. En muchos de los lazaretos no existían construcciones concretas para la vigilancia, por lo que se ejercía mediante la colocación de guardias en sus diferentes recintos. En el Vecchio de Venecia, por ejemplo, se practicaba con la presencia de un guardia asignado a cada uno de los grupos en cuarentena, de los que se cuidaba en todo momento ${ }^{56}$. Me centraré en dos tipos de configuración de los que se desprende una voluntad de vigilancia a través de propuestas arquitectónicas concretas.

El primer tipo de distribución buscaba una vigilancia del lazareto desde un punto central. Esta idea nos remite, inevitablemente, a otros edificios de encierro: al hospital y la casa de misericordia renacentistas de planta cruciforme ${ }^{57}$, a los edificios estrellados utilizados como hospitales y prisiones

54. Hély d'Oissel, n. 15, p. 17; Rodríguez, Manuel. Lazareto de Maón. Mahón: Imp. de Pedro Antonio Serra; 1813, p. 12.

55. Howard, n. 33, p. 23. En el informe Hély d'Oissel se recomienda ardientemente que, dentro de un orden, los cuarentenistas tengan una estancia en el lazareto lo más agradable y divertida posible, Hély d'Oissel, n. 15, p. 25-26. Una de las orientaciones de la arquitectura presidiaria de la época iba en el sentido de comunicar, desde el exterior del edificio, la severidad que esperaba a los presos en su interior, Pevsner, Nikolaus. Historia de las tipologías arquitectónicas. Barcelona: Gustavo Gili; 1979, p. 192-194. Véase también McGowen, Randall. The wellordered prison. England, 1780-1865. In: Morris, Norvall; Rothman, David. The Oxford history of the prison. Oxford: OUP; 1995, p. 79-109; Fraile, Pedro. Un espacio para castigar. La cárcel y la ciencia penitenciaria en España (siglos XVIII-XIX). Barcelona: Ediciones del Serbal; 1987.

56. Howard, n. 33, p. 16.

57. Fraile, Pedro. El vigilante de la atalaya. La génesis de los espacios de control en los albores del capitalismo. Lleida: Editorial Milenio; 2005. 
a partir del del siglo XVIII y a las cárceles basadas en el modelo panóptico teorizado por Bentham a finales del mismo siglo ${ }^{58}$.

En muchos casos, se trataba solamente de un centro simbólico que articulaba el complejo y sobre el que, ineludiblemente, confluían las miradas. Solía estar ocupado por una capilla que servia para escenificar el poder, como vemos en los lazaretos renacentistas de Milán, Bérgamo o Verona. Desde dichas capillas se oficiaba la misa para los confinados y el orden divino se superponía al temporal, así como el orden cuarentenario se superponía a la vida exterior. Este efecto lo vemos también en otros lazaretos de líneas menos geométricas, como el de Mesina o el de La Spezia, en los cuales la capilla ocupa un espacio más o menos central. En todos ellos la vigilancia se ejercía desde el patio central hacia fuera, donde estaban situadas las celdas de los cuarentenistas.

En otras ocasiones este control sobre el lazareto se practicaba realmente desde un torreón que ocupaba un lugar privilegiado en el recinto. El ejemplo más puro de esta voluntad fiscalizadora no pasó del estadio de proyecto. Se trata de un lazareto contra el cólera planeado para la localidad siciliana de San Michele, cerca de Caltagirone ${ }^{59}$, fechado en 1837. Debía tener una forma redonda y las estancias para los confinados debían disponerse a lo largo del muro de cinta. En el centro del patio, y presidiendo todo el complejo, se situaría una torre de dos pisos consagrada al cuerpo de guardia. Las similitudes con el panóptico benthamita, que se perciben de manera más que evidente en el plano, hacen innecesario cualquier comentario.

El segundo tipo de configuración en la cual la vigilancia desempeñaba un papel importante la presentan los lazaretos en pabellones. En este tipo de lazareto se llevó a cabo una reestructuración del espacio cuarentenario aplicando una estricta clasificación de confinados y mercancías en áreas compartimentadas y estancas. Esta reordenación se hizo teniendo en cuenta la organización de las operaciones desarrolladas en el recinto y su vigilancia. Esto comportaba una distribución del recinto basada en un minucioso estudio de los trayectos a realizar en su interior, de la peligrosidad de sus ocupantes y de su vigilancia. Todo ello se articulaba desde las dependen-

58. Bentham, Jeremy. Panopticon; or the inspection house. In: Bowring, John. The works of Jeremy Bentham. Published under the superintendence of his executor..., vol. IV. Edimburgo: William Tait; 1843, p. 38-172.

59. Agradezco al profesor Mario Alberghina, de la Universidad de Catania, por haberme enviado una copia del plano de este lazareto. 


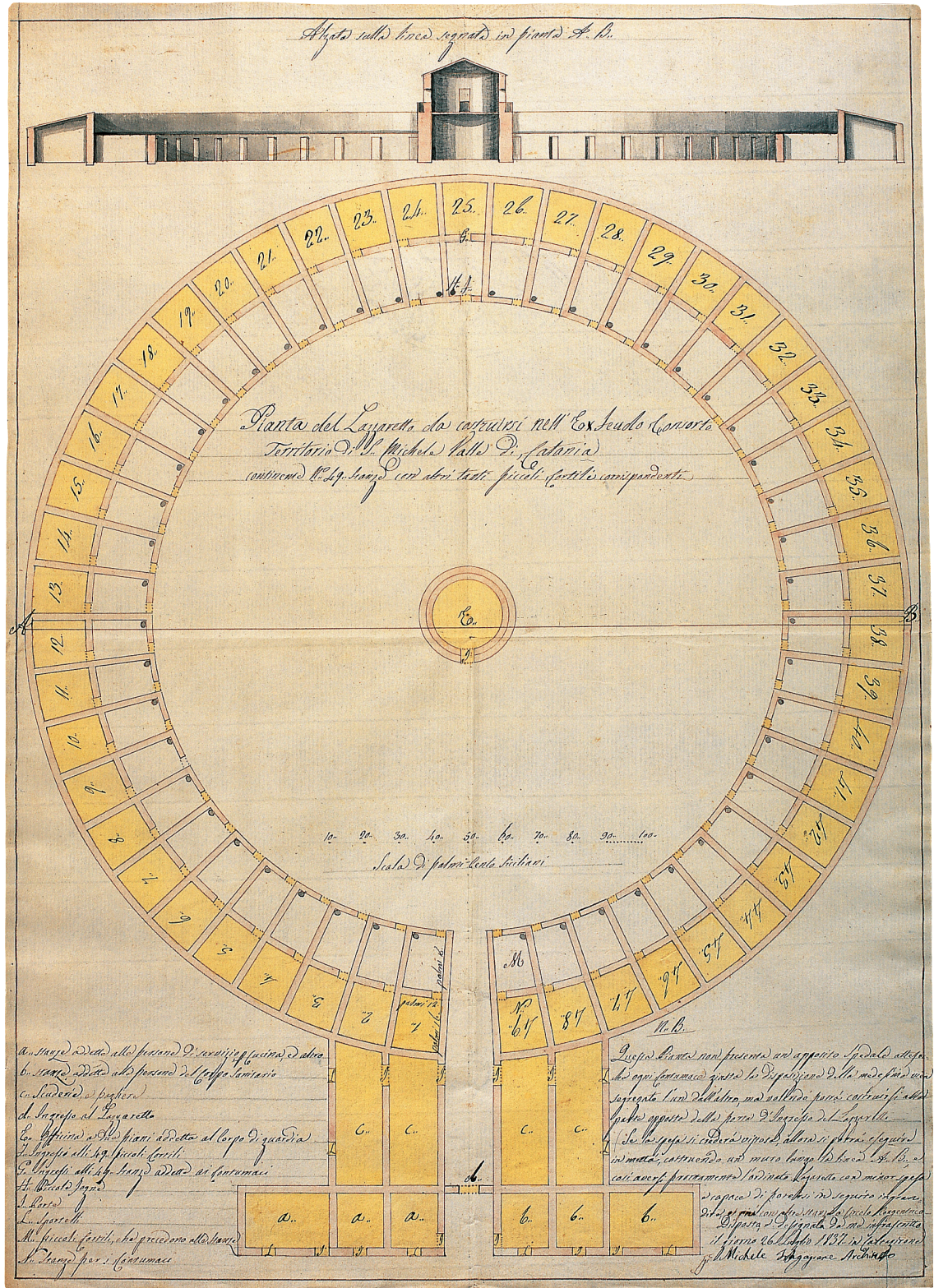

Figura 4: Proyecto de Lazareto para San Michele en la primera mitad del siglo XIX. Fuente: Copia cedida por el Prof. Mario Alberghina de la Universidad de Catania. 


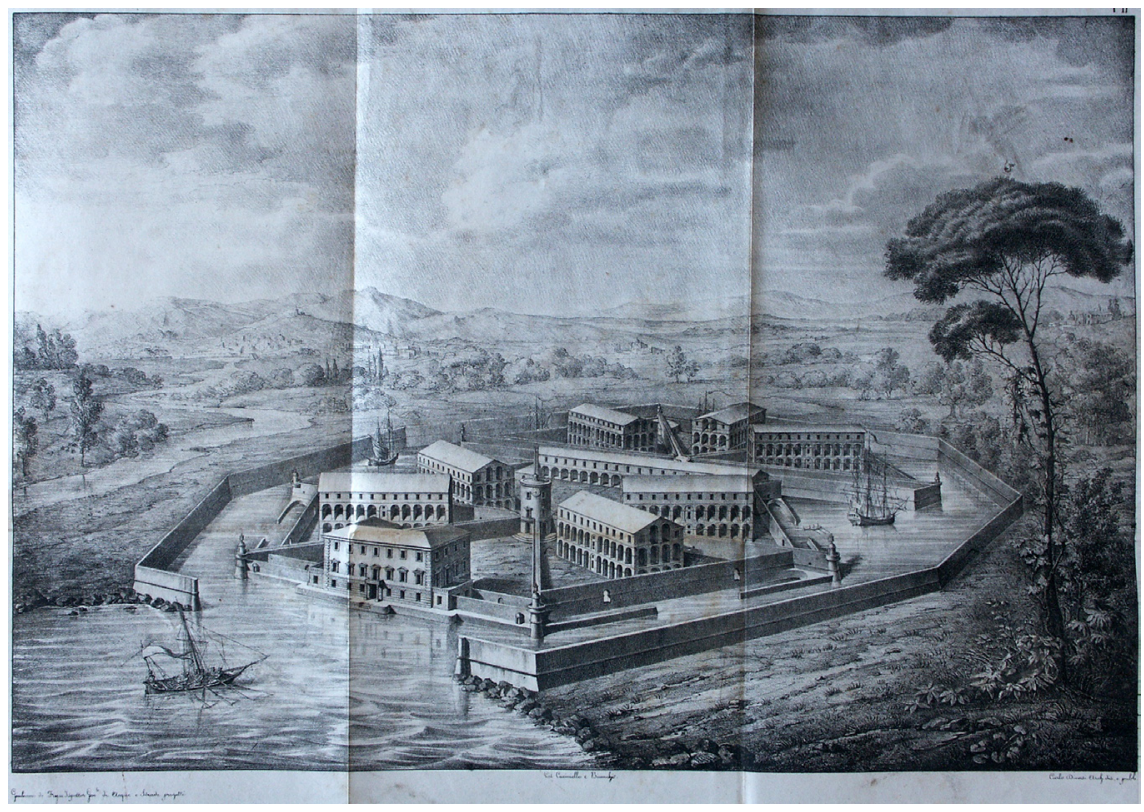

Figura 5: Vista del proyecto de lazareto para las cercanías de Nápoles en la primera mitad del siglo XIX. Fuente: De Fazio, Giuliano. Sistema Generale dell' Archittertura de Lazzeretti. Nápoles: Angelo Trani; 1826, tabla II.

cias del capitán, un punto más o menos céntrico desde donde se pudiera ejercer un control sobre todo el lazareto. Con todo, no debemos entender esta casa del capitán como un lugar desde el cual se ejercía una vigilancia total del lazareto, sino más bien como un centro de operaciones desde donde el capitán organizaba la vida en su interior. La verdadera vigilancia se ejercía de manera compartimentada desde los ángulos de cada uno de los módulos del lazareto. La adopción del cuadrado como unidad básica permitía una mayor vigilancia con un menor número de guardias ${ }^{60}$, que ejercerían una vigilancia centrípeta.

Acabaremos con un ejemplo en el que confluyen los dos modelos de vigilancia mencionados. Se trata de un proyecto de lazareto para Mesina, concebido por Pompeo Schianterelli hacia 1799. El diseño fue retomado dos 
décadas más tarde por Giuliano de Fazio, discípulo del anterior y encargado de concebir una estación de cuarentena para Nápoles. El interés principal de ambos proyectos reside en que se trata de lazaretos con una ordenación pabellonaria y una disposición panóptica. Esta combinación la permitía su forma poligonal, un octógono y un hexágono respectivamente.

El principio sobre el que se fundaba la vigilancia de estos lazaretos era que sus oficiales debían poder pasear por todo el complejo sin peligro para su salud. En el centro del hexágono había una torre de vigía en cuyo tercer piso estaba instalada la capilla, y en el segundo la sala de guardia central. Desde ésta última se podía ejercer un control panóptico de todo el hexágono y, además, se accedía al deambulatorio colocado sobre los muros que cerraban cada patio y que servía para la ronda de los guardias. En el extremo de cada uno de los muros radiales había una garita ${ }^{61}$. Esta disposición permitía, a la vez, la vigilancia centrípeta típica de los lazaretos pabellonarios y la centrífuga propia de las instituciones panópticas ${ }^{62}$.

61. De Fazio, Giuliano. Sistema generale dell'archittertura de lazzeretti. Nápoles: Angelo Trani; 1826, p. 16.

62. Bonastra, Quim. Le lazaret, à la croisée de traditions architecturales hétérogènes. Revue de la Société Française d'Histoire des Hôpitaux. 2008; 131-132: 65-69; Fraile, n. 57, p. 191-226. 